\title{
Synthese und Struktur des Trifluoracetyldicyanomethanids
}

\author{
Synthesis and Structure of Trifluoroacetyldicyanomethanide \\ Herbert W. Roesky*, Mathias Noltemeyer und George M. Sheldrick \\ Institut für Anorganische Chemie der Universität, Tammannstraße 4, D-3400 Göttingen, FRG \\ Z. Naturforsch. 40b, 883-885 (1985); eingegangen am 7. März 1985 \\ X-Ray, Carbanion, Fluorine, Nitrile \\ A suspension of $\mathrm{NaCN}$ in $\mathrm{CH}_{3} \mathrm{CN}$ was treated with excess of $\mathrm{C}_{2} \mathrm{~F}_{4}$. The resulting red \\ solution was evaporated to dryness and a red amorphous solid remained. One part of \\ this solid was dissolved in water and a solution of $\mathrm{Ph}_{4} \mathrm{AsCl}$ was added. During the \\ work-up procedure two salts were isolated, yellow $\left[\mathrm{Ph}_{4} \mathrm{As}^{+}\right]\left[\mathrm{CF}_{3} \mathrm{C}(\mathrm{O}) \mathrm{C}(\mathrm{CN})_{2}^{-}\right] \mathbf{1} \mathbf{a}$ and red \\ $\left[\mathrm{Ph}_{4} \mathrm{As}^{+}\right]\left[(\mathrm{NC})_{2} \mathrm{CC}(\mathrm{CN}) \mathrm{C}(\mathrm{F}) \mathrm{NC}(\mathrm{CN})_{2}{ }^{-}\right] \mathbf{2} \mathbf{a}$. The products were characterized on the basis of field \\ desorption mass spectroscopy for positive and negative ions. 1a was further characterized by an \\ $\mathrm{X}$-ray single crystal investigation.
}

In einer Reihe von Arbeiten konnten wir zeigen, daß Hexafluoraceton mit Cyaniden zu interessanten Cycloadditionsprodukten führt [1,2]. Verwendet man anstelle von Hexafluoraceton Dicyan und Kaliumcyanid, so reagieren diese Verbindungen zu dem Anion $\left[\mathrm{C}_{7} \mathrm{~N}_{7}^{-}\right]$. Aus spektroskopischen Untersuchungen wird dem Anion eine bicyclische Struktur zugeordnet [3]. Eine Röntgenstrukturanalyse an $\left[\mathrm{Ph}_{3} \mathrm{PNPPh}_{3}{ }^{+}\right]\left[\mathrm{C}_{7} \mathrm{~N}_{7}{ }^{-}\right]$konnte dies bestätigen [4].

In diesem Beitrag berichten wir über die Reaktion von Tetrafluorethen mit Natriumcyanid in Acetonitril, die nach Aufarbeiten in Wasser zu den Anionen Trifluoracetyldicyanmethanid $\mathbf{1}$ und 3-Fluoro1,1,4,5,5-pentacyano-2-azapentadienid 2 führt.

\section{Experimenteller Teil}

${ }^{19}$ F-NMR-Spektrum: Bruker WP 80 SY $(75,39 \mathrm{MHz}), \mathrm{CFCl}_{3}$ als interner Standard. - IRSpektren: Perkin-Elmer-Spektrograph 735 B.

Tetraphenylarsonium-trifluoracetyldicyanomethanid (1a) und Tetraphenylarsonium-3-fluoro-

\section{1,1,4,5,5-pentacyano-2-azapentadienid (2a)}

$16 \mathrm{~g} \mathrm{NaCN}$ werden in $300 \mathrm{ml} \mathrm{CH}_{3} \mathrm{CN}$ suspendiert und auf $30-40{ }^{\circ} \mathrm{C}$ erwärmt. Dann leitet man innerhalb von 3,5 h $100 \mathrm{~g} \mathrm{C}_{2} \mathrm{~F}_{4}$ langsam durch die Lösung. Nicht umgesetztes $\mathrm{C}_{2} \mathrm{~F}_{4}$ wird in einer Kondensationsfalle aufgefangen. Danach wird die rote Reaktionslösung von nicht umgesetztem $\mathrm{NaCN}$ abfiltriert und aus dem Filtrat das $\mathrm{CH}_{3} \mathrm{CN}$ i. Vak. abgezogen. Es bleibt ein roter amorpher Feststoff zurück $(5,0 \mathrm{~g})$. Etwa $0,1 \mathrm{~g}$ des Rohproduktes wird in $100 \mathrm{ml}$ Wasser

* Sonderdruckanforderungen an Prof. Dr. H. W Roesky. Verlag der Zeitschrift für Naturforschung, D-7400 Tübingen 0340-5087/85/0700-0883/\$ 01.00/0 gelöst, dabei entsteht eine dunkelrote Lösung. $\mathrm{Zu}$ dieser Lösung gibt man $0,4 \mathrm{~g} \mathrm{Ph}_{4} \mathrm{AsCl}$ in $100 \mathrm{ml} \mathrm{H}_{2} \mathrm{O}$ gelöst. Es entsteht ein roter Niederschlag, der sich innerhalb von $14 \mathrm{~d}$ absetzt und durch Filtration gewonnen werden kann. Anschließend wird der Niederschlag mit $200 \mathrm{ml} \mathrm{C}_{2} \mathrm{H}_{5} \mathrm{OH}$ behandelt, dabei geht die Hälfte in Lösung. Zu der Ethanollösung gibt man die gleiche Menge Wasser hinzu und läßt bei R.T. stehen. Es scheiden sich nach 5-6 Wochen gelbe kristalline Nadeln von 1a ab. Ausb. $50 \mathrm{mg}$.

Der nach der Ethanolbehandlung verbleibende Rückstand wird in Aceton gelöst und die gleiche Menge Wasser zugegeben. Nach 3 Wochen scheiden sich rote Kristallnadeln von 2a ab. Ausb. $0,8 \mathrm{~g}$.

1a: ${ }^{19} \mathrm{~F}-\mathrm{NMR}\left(\left(\mathrm{CD}_{3}\right)_{2} \mathrm{CO}\right): \delta=-72,1,{ }^{1} J_{\mathrm{C}-\mathrm{F}}=$ $291 \mathrm{~Hz} .-$ Schmp. $96-102{ }^{\circ} \mathrm{C}$. $-\mathrm{MS}(-\mathrm{FD}): \mathrm{m} / z=$ $161\left(\mathrm{CF}_{3} \mathrm{C}(\mathrm{O}) \mathrm{C}(\mathrm{CN})_{2}\right),(+\mathrm{FD}) 383\left(\mathrm{Ph}_{4} \mathrm{As}\right) .-$ IR: $v(\mathrm{C} \equiv \mathrm{N}) 2216$ und 2202, $v(\mathrm{CO}) 1612$.

2a: MS (-FD): $m / z 211$

$\left[(\mathrm{CN})_{2} \mathrm{CC}(\mathrm{CN}) \mathrm{C}(\mathrm{F}) \mathrm{NC}(\mathrm{CN})_{2}\right],(+\mathrm{FD}) 383\left(\mathrm{Ph}_{4} \mathrm{As}\right)$.

\section{Experimentelles zur Röntgenstrukturanalyse}

4976 Reflexe wurden im Bereich $2 \theta<45^{\circ}$ mit $\operatorname{MoK}_{\alpha}$-Strahlung $(\lambda=71,069 \mathrm{pm})$ von einem Kristall $0,04 \times 0,2 \times 0,6 \mathrm{~mm}^{3}$ auf einem Stoe-Siemens-Vierkreisdiffraktometer vermessen. Die Struktur ließ sich mit automatischen direkten Methoden lösen und wurde mit allen Nicht-H-Atomen anisotrop bis $R=$ $0,065, R_{w}=0,058\left(\right.$ Gewichtsschema $\mathrm{w}^{-1}=\sigma^{2}(\mathrm{~F})+$ $\left.0,0006 \mathrm{~F}^{2}\right)$ für 2460 gemittelte Reflexe mit $\mathrm{F}>3 \sigma(\mathrm{F})$ verfeinert. Alle Berechnungen erfolgten auf einem Eclipse S-250-Minirechner mit dem SHELXTL-Programmsystem von G.M.S.

\section{Ergebnisse}

Die Reaktion von Tetrafluorethen mit $\mathrm{Na}-$ triumcyanid in Acetonitril führt zu einem inten- 
siv rot gefärbten Produktgemisch. Durch Aufarbeiten in wäßriger Lösung erhält man daraus die Anionen $\left[\mathrm{CF}_{3} \mathrm{C}(\mathrm{O}) \mathrm{C}(\mathrm{CN})_{2}^{-}\right]$(1) und $\left[(\mathrm{NC})_{2} \mathrm{CC}(\mathrm{CN}) \mathrm{C}(\mathrm{F}) \mathrm{NC}(\mathrm{CN})_{2}^{-}\right]$(2). Die Anionen wurden als Tetraphenylarsoniumsalze isoliert. $\left[\mathrm{Ph}_{4} \mathrm{As}^{+}\right]\left[\mathrm{CF}_{3} \mathrm{C}(\mathrm{O}) \mathrm{C}(\mathrm{CN})_{2}{ }^{-}\right](\mathbf{1 a})$ ist eine gelbe und $\left[\mathrm{Ph}_{4} \mathrm{As}^{+}\right]\left[(\mathrm{NC})_{2} \mathrm{CC}(\mathrm{CN}) \mathrm{C}(\mathrm{F}) \mathrm{NC}(\mathrm{CN})_{2}{ }^{-}\right] \quad$ (2a) eine rote Verbindung.

Das Anion 2 ist bekannt. Es entsteht aus Natriumcyanid und 1,2-Dichlorhexafluorcyclopenten [5] nach einem nicht bekannten Mechanismus; die Struktur wurde durch eine Röntgenstrukturanalyse [6] geklärt. Die Bildungstendenz für $\mathbf{2}$ ist offensichtlich so groß, daß ungesättigte $\mathrm{CF}-$ Verbindungen und Cyanidionen leicht zu dem 3-Fluoro-1,1,4,5,5-pentacyano-2-azapentadienid führen. Wir konnten $2 \mathbf{a}$ anhand des Felddesorptionsspektrums für negative und

Tab. I. Atomkoordinaten $\left(\times 10^{4}\right)$.

\begin{tabular}{|c|c|c|c|}
\hline & $x$ & $y$ & $z$ \\
\hline $\operatorname{As}(1)$ & 5000 & $5530(1)$ & 2500 \\
\hline $\mathrm{C}(11)$ & $4418(4)$ & $7045(8)$ & $1822(3)$ \\
\hline$C(12)$ & $4743(4)$ & $8337(8)$ & $1471(4)$ \\
\hline $\mathrm{C}(13)$ & $4335(4)$ & $9507(8)$ & $1016(4)$ \\
\hline $\mathrm{C}(14)$ & $3610(4)$ & $9370(8)$ & $924(4)$ \\
\hline$C(15)$ & $3292(4)$ & $8094(9)$ & $1277(4)$ \\
\hline$C(16)$ & $3702(4)$ & $6889(8)$ & $1733(4)$ \\
\hline $\mathrm{C}(21)$ & $5552(3)$ & $4032(7)$ & $1960(3)$ \\
\hline $\mathrm{C}(22)$ & $5556(3)$ & 4188(8) & 1179(4) \\
\hline $\mathrm{C}(23)$ & $5914(4)$ & 2993(8) & $810(4)$ \\
\hline$C(24)$ & $6264(3)$ & $1663(8)$ & $1207(4)$ \\
\hline $\mathrm{C}(25)$ & $6265(4)$ & $1499(8)$ & 1981(4) \\
\hline$C(26)$ & 5901(3) & $2697(7)$ & $2368(4)$ \\
\hline $\operatorname{As}(2)$ & 0 & $5232(1)$ & 2500 \\
\hline$C(31)$ & $706(3)$ & $3771(8)$ & $3014(3)$ \\
\hline $\mathrm{C}(32)$ & $1410(4)$ & $4100(9)$ & 3011(4) \\
\hline$C(33)$ & 1918(4) & 2992(12) & $3384(5)$ \\
\hline$C(34)$ & $1704(4)$ & 1511(11) & $3715(5)$ \\
\hline$C(35)$ & 999(4) & $1155(9)$ & $3712(4)$ \\
\hline$C(36)$ & $500(4)$ & $2295(8)$ & $3360(3)$ \\
\hline$C(41)$ & 391(3) & $6700(7)$ & $1795(3)$ \\
\hline $\mathrm{C}(42)$ & $870(4)$ & $7948(8)$ & $2090(4)$ \\
\hline C(43) & $1146(4)$ & $9071(10)$ & $1602(4)$ \\
\hline$C(44)$ & $927(4)$ & 8941(10) & $831(4)$ \\
\hline$C(45)$ & 441(4) & $7745(10)$ & $548(4)$ \\
\hline $\mathrm{C}(46)$ & $175(4)$ & $6584(8)$ & $1023(3)$ \\
\hline $\mathrm{C}(1)$ & $7325(4)$ & $3138(8)$ & $5393(5)$ \\
\hline $\mathrm{C}(2)$ & $7273(4)$ & $2765(9)$ & $6166(5)$ \\
\hline $\mathrm{N}(1)$ & $7242(4)$ & $2433(11)$ & $6799(4)$ \\
\hline$C(3)$ & $6694(4)$ & $3490(8)$ & $4905(4)$ \\
\hline $\mathrm{N}(2)$ & $6167(3)$ & $3759(9)$ & $4508(4)$ \\
\hline$C(4)$ & $7990(4)$ & $3062(8)$ & $5172(5)$ \\
\hline$C(5)$ & $8062(4)$ & $3523(10)$ & $4339(5)$ \\
\hline $\mathrm{O}(1)$ & $8549(3)$ & $2695(7)$ & $5583(4)$ \\
\hline $\mathrm{F}(1)$ & $7879(4)$ & $5054(8)$ & 4151(4) \\
\hline $\mathrm{F}(2)$ & $8706(3)$ & $3334(7)$ & $4175(4)$ \\
\hline $\mathrm{F}(3)$ & $7670(3)$ & $2505(10)$ & $3846(3)$ \\
\hline
\end{tabular}

positive Ionen eindeutig charakterisieren. Die Eigenschaften stimmen mit dem von Carpenter et al. [5] gefundenen Produkt gut überein. Der Einbau des Sauerstoffes in $\mathbf{1}$ muß durch das Wasser erfolgen. Es war deshalb naheliegend, $\mathbf{1}$ auf anderem Wege herzustellen; dazu wurde Malonsäuredinitril mit Natriumethylat und Trifluoracetylchlorid umgesetzt:

$$
\begin{aligned}
& \mathrm{H}_{2} \mathrm{C}(\mathrm{CN})_{2}+2 \mathrm{NaOC}_{2} \mathrm{H}_{5}+\mathrm{CF}_{3} \mathrm{C}(\mathrm{O}) \mathrm{Cl} \longrightarrow \\
& {\left[\mathrm{CF}_{3} \mathrm{C}(\mathrm{O}) \mathrm{C}(\mathrm{CN})_{2}{ }^{-}\right] \mathrm{Na}^{+}+\mathrm{NaCl}+2 \mathrm{C}_{2} \mathrm{H}_{5} \mathrm{OH}}
\end{aligned}
$$

Bei dieser Umsetzung lie $\beta$ sich 1 nicht nachweisen, es entstehen dabei hauptsächlich $\mathrm{CF}_{3} \mathrm{C}(\mathrm{O})-\mathrm{OC}_{2} \mathrm{H}_{5}$ und Natriumchlorid.

Die Charakterisierung von 1a erfolgte durch ein Felddesorptionsmassenspektrum und durch eine Einkristallröntgenstrukturanalyse. Für negative Ionen findet $\operatorname{man}$ bei $\mathrm{m} / \mathrm{z}=161$ das Anion $\left[\mathrm{CF}_{3} \mathrm{C}(\mathrm{O}) \mathrm{C}(\mathrm{CN})_{2}^{-}\right]$und keine weiteren Ionen.

\section{Kristallstrukturvon $\left.\left[\mathrm{Ph}_{4} \mathrm{As}^{+}\right] / \mathrm{CF}_{3} \mathrm{C}(\mathrm{O}) \mathrm{C}(\mathrm{CN})_{2}^{-}\right](\mathbf{1 a})$}

1 a kristallisiert monoklin in der Raumgruppe $\mathrm{P} 2 / \mathrm{c}$ mit $a=1905,8(4), b=778,6(2), c=1760,4(4) \mathrm{pm}$ und $\beta=97,97(2)^{\circ}$. Mit vier Formeleinheiten/Elementarzelle ergab sich $\mathrm{D}_{x}=1,398 \mathrm{~g} \mathrm{~cm}^{-3}$ und $\mu\left(\mathrm{MoK}_{\alpha}\right)=1,35 \mathrm{~mm}^{-1}$. Atomkoordinaten, Bindungslängen und -winkel sind in Tabn. I-III angegeben; die Struktur des Anions in 1a wird in Abb. 1 gezeigt. Zwei unabhängige Kationen liegen auf zweizähligen Achsen; das Anion besitzt eine allgemeine Lage. Außer $F(1)$ und $F(3)$ liegen alle Atome in

Tab. II. Bindungslängen (pm).

\begin{tabular}{llll}
\hline As(1)-C(11) & $191,8(6)$ & As(1)-C(21) & $190,9(7)$ \\
As(1)-C(11a) & $191,8(6)$ & As(1)-C(21a) & $190,9(7)$ \\
$\mathrm{C}(11)-C(12)$ & $137,3(9)$ & $\mathrm{C}(11)-\mathrm{C}(16)$ & $135,8(10)$ \\
$\mathrm{C}(12)-\mathrm{C}(13)$ & $138,0(9)$ & $\mathrm{C}(13)-\mathrm{C}(14)$ & $137,4(11)$ \\
$\mathrm{C}(14)-\mathrm{C}(15)$ & $135,8(10)$ & $\mathrm{C}(15)-\mathrm{C}(16)$ & $140,0(9)$ \\
$\mathrm{C}(21)-\mathrm{C}(22)$ & $138,3(9)$ & $\mathrm{C}(21)-\mathrm{C}(26)$ & $138,1(8)$ \\
$\mathrm{C}(22)-\mathrm{C}(23)$ & $136,9(10)$ & $\mathrm{C}(23)-\mathrm{C}(24)$ & $137,0(9)$ \\
$\mathrm{C}(24)-\mathrm{C}(25)$ & $136,9(10)$ & $\mathrm{C}(25)-\mathrm{C}(26)$ & $139,5(9)$ \\
$\mathrm{As}(2)-\mathrm{C}(31)$ & $189,4(6)$ & $\mathrm{As}(2)-\mathrm{C}(41)$ & $191,3(6)$ \\
$\mathrm{As}(2)-\mathrm{C}(31 \mathrm{a})$ & $189,5(6)$ & $\mathrm{As}(2)-\mathrm{C}(41 \mathrm{a})$ & $191,3(6)$ \\
$\mathrm{C}(31)-\mathrm{C}(32)$ & $136,7(9)$ & $\mathrm{C}(31)-\mathrm{C}(36)$ & $138,3(9)$ \\
$\mathrm{C}(32)-\mathrm{C}(33)$ & $139,1(11)$ & $\mathrm{C}(33)-\mathrm{C}(34)$ & $137,9(13)$ \\
$\mathrm{C}(34)-\mathrm{C}(35)$ & $137,0(11)$ & $\mathrm{C}(35)-\mathrm{C}(36)$ & $138,3(9)$ \\
$\mathrm{C}(41)-\mathrm{C}(42)$ & $138,4(9)$ & $\mathrm{C}(41)-\mathrm{C}(46)$ & $136,7(8)$ \\
$\mathrm{C}(42)-\mathrm{C}(43)$ & $137,9(10)$ & $\mathrm{C}(43)-\mathrm{C}(44)$ & $136,7(10)$ \\
$\mathrm{C}(44)-\mathrm{C}(45)$ & $135,9(11)$ & $\mathrm{C}(45)-\mathrm{C}(46)$ & $137,6(10)$ \\
$\mathrm{C}(1)-\mathrm{C}(2)$ & $140,7(13)$ & $\mathrm{C}(1)-\mathrm{C}(3)$. & $140,5(10)$ \\
$\mathrm{C}(1)-\mathrm{C}(4)$ & $137,8(11)$ & $\mathrm{C}(2)-\mathrm{N}(1)$ & $115,3(12)$ \\
$\mathrm{C}(3)-\mathrm{N}(2)$ & $116,1(9)$ & $\mathrm{C}(4)-\mathrm{C}(5)$ & $153,5(13)$ \\
$\mathrm{C}(4)-\mathrm{O}(1)$ & $123,5(9)$ & $\mathrm{C}(5)-\mathrm{F}(1)$ & $127,4(10)$ \\
$\mathrm{C}(5)-\mathrm{F}(2)$ & $130,6(10)$ & $\mathrm{C}(5)-\mathrm{F}(3)$ & $132,6(10)$ \\
\hline
\end{tabular}




\begin{tabular}{llll}
\hline $\mathrm{C}(11)-\mathrm{As}(1)-\mathrm{C}(21)$ & $112,1(3)$ & $\mathrm{C}(11)-\mathrm{As}(1)-\mathrm{C}(11 \mathrm{a})$ & $104,1(4)$ \\
$\mathrm{C}(21)-\mathrm{As}(1)-\mathrm{C}(11 \mathrm{a})$ & $112,0(3)$ & $\mathrm{C}(11)-\mathrm{As}(1)-\mathrm{C}(21 \mathrm{a})$ & $112,0(3)$ \\
$\mathrm{C}(21)-\mathrm{As}(1)-\mathrm{C}(21 \mathrm{a})$ & $104,7(4)$ & $\mathrm{As}(1)-\mathrm{C}(11)-\mathrm{C}(12)$ & $118,2(5)$ \\
$\mathrm{As}(1)-\mathrm{C}(11)-\mathrm{C}(16)$ & $120,1(5)$ & $\mathrm{C}(12)-\mathrm{C}(11)-\mathrm{C}(16)$ & $121,5(6)$ \\
$\mathrm{C}(11)-\mathrm{C}(12)-\mathrm{C}(13)$ & $119,5(7)$ & $\mathrm{C}(12)-\mathrm{C}(13)-\mathrm{C}(14)$ & $119,6(7)$ \\
$\mathrm{C}(13)-\mathrm{C}(14)-\mathrm{C}(15)$ & $120,6(6)$ & $\mathrm{C}(14)-\mathrm{C}(15)-\mathrm{C}(16)$ & $120,2(7)$ \\
$\mathrm{C}(11)-\mathrm{C}(16)-\mathrm{C}(15)$ & $118,6(6)$ & $\mathrm{As}(1)-\mathrm{C}(21)-\mathrm{C}(22)$ & $121,4(4)$ \\
$\mathrm{As}(1)-\mathrm{C}(21)-\mathrm{C}(26)$ & $117,4(5)$ & $\mathrm{C}(22)-\mathrm{C}(21)-\mathrm{C}(26)$ & $121,0(6)$ \\
$\mathrm{C}(21)-\mathrm{C}(22)-\mathrm{C}(23)$ & $119,0(6)$ & $\mathrm{C}(22)-\mathrm{C}(23)-\mathrm{C}(24)$ & $120,8(6)$ \\
$\mathrm{C}(23)-\mathrm{C}(24)-\mathrm{C}(25)$ & $120,8(6)$ & $\mathrm{C}(24)-\mathrm{C}(25)-\mathrm{C}(26)$ & $119,5(6)$ \\
$\mathrm{C}(21)-\mathrm{C}(26)-\mathrm{C}(25)$ & $119,1(6)$ & $\mathrm{C}(31)-\mathrm{As}(2)-\mathrm{C}(41)$ & $110,7(3)$ \\
$\mathrm{C}(31)-\mathrm{As}(2)-\mathrm{C}(31 \mathrm{a})$ & $106,2(4)$ & $\mathrm{C}(41)-\mathrm{As}(2)-\mathrm{C}(31 \mathrm{a})$ & $111,4(3)$ \\
$\mathrm{C}(31)-\mathrm{As}(2)-\mathrm{C}(41 \mathrm{a})$ & $111,4(3)$ & $\mathrm{C}(41)-\mathrm{As}(2)-\mathrm{C}(41 \mathrm{a})$ & $106,6(4)$ \\
$\mathrm{As}(2)-\mathrm{C}(31)-\mathrm{C}(32)$ & $121,2(5)$ & $\mathrm{As}(2)-\mathrm{C}(31)-\mathrm{C}(36)$ & $118,9(5)$ \\
$\mathrm{C}(32)-\mathrm{C}(31)-\mathrm{C}(36)$ & $119,8(6)$ & $\mathrm{C}(31)-\mathrm{C}(32)-\mathrm{C}(33)$ & $120,1(7)$ \\
$\mathrm{C}(32)-\mathrm{C}(33)-\mathrm{C}(34)$ & $119,4(8)$ & $\mathrm{C}(33)-\mathrm{C}(34)-\mathrm{C}(35)$ & $121,0(7)$ \\
$\mathrm{C}(34)-\mathrm{C}(35)-\mathrm{C}(36)$ & $119,0(7)$ & $\mathrm{C}(31)-\mathrm{C}(36)-\mathrm{C}(35)$ & $120,7(6)$ \\
$\mathrm{As}(2)-\mathrm{C}(41)-\mathrm{C}(42)$ & $118,2(5)$ & $\mathrm{As}(2)-\mathrm{C}(41)-\mathrm{C}(46)$ & $120,9(5)$ \\
$\mathrm{C}(42)-\mathrm{C}(41)-\mathrm{C}(46)$ & $120,8(6)$ & $\mathrm{C}(41)-\mathrm{C}(42)-\mathrm{C}(43)$ & $120,0(6)$ \\
$\mathrm{C}(42)-\mathrm{C}(43)-\mathrm{C}(44)$ & $118,7(7)$ & $\mathrm{C}(43)-\mathrm{C}(44)-\mathrm{C}(45)$ & $120,9(7)$ \\
$\mathrm{C}(44)-\mathrm{C}(45)-\mathrm{C}(46)$ & $121,1(7)$ & $\mathrm{C}(41)-\mathrm{C}(46)-\mathrm{C}(45)$ & $118,4(6)$ \\
$\mathrm{C}(2)-\mathrm{C}(1)-\mathrm{C}(3)$ & $117,5(7)$ & $\mathrm{C}(2)-\mathrm{C}(1)-\mathrm{C}(4)$ & $117,1(7)$ \\
$\mathrm{C}(3)-\mathrm{C}(1)-\mathrm{C}(4)$ & $125,4(8)$ & $\mathrm{C}(1)-\mathrm{C}(2)-\mathrm{N}(1)$ & $178,5(9)$ \\
$\mathrm{C}(1)-\mathrm{C}(3)-\mathrm{N}(2)$ & $178,8(8)$ & $\mathrm{C}(1)-\mathrm{C}(4)-\mathrm{C}(5)$ & $117,9(7)$ \\
$\mathrm{C}(1)-\mathrm{C}(4)-\mathrm{O}(1)$ & $126,7(8)$ & $\mathrm{C}(5)-\mathrm{C}(4)-\mathrm{O}(1)$ & $115,4(7)$ \\
$\mathrm{C}(4)-\mathrm{C}(5)-\mathrm{F}(1)$ & $114,1(7)$ & $\mathrm{C}(4)-\mathrm{C}(5)-\mathrm{F}(2)$ & $113,3(7)$ \\
$\mathrm{F}(1)-\mathrm{C}(5)-\mathrm{F}(2)$ & $106,3(7)$ & $\mathrm{C}(4)-\mathrm{C}(5)-\mathrm{F}(3)$ & $111,6(7)$ \\
$\mathrm{F}(1)-\mathrm{C}(5)-\mathrm{F}(3)$ & $106,3(7)$ & $\mathrm{F}(2)-\mathrm{C}(5)-\mathrm{F}(3)$ & $104,5(7)$ \\
& & &
\end{tabular}

Tab. III. Bindungswinkel $\left(^{\circ}\right)$.

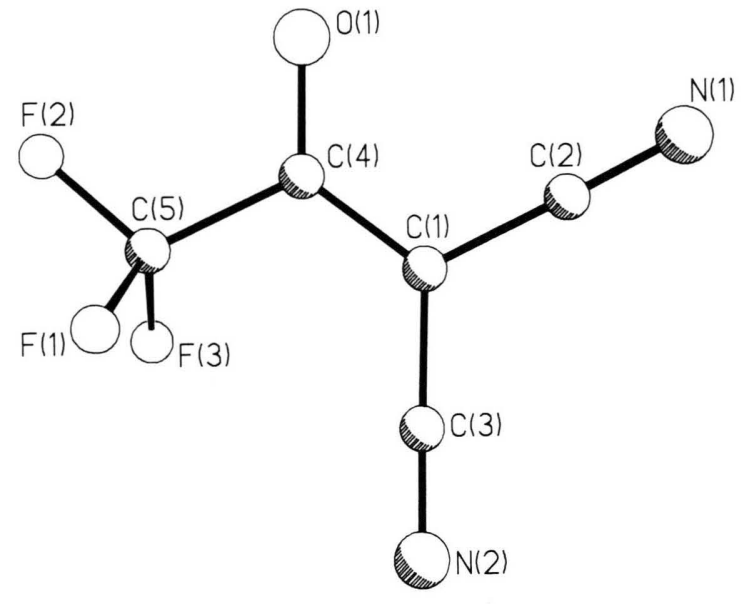

Abb. 1. Struktur des Anions in 1a mit Atombezeichnung. einer Ebene (mittlere Abweichung 2,8 pm). Eine Librationsanalyse zeigte, daß die endständigen $\mathrm{C} \equiv \mathrm{N}$ und $\mathrm{C}-\mathrm{F}$-Bindungen erheblich $(2-6 \mathrm{pm})$ verkürzt sind. Die Struktur ist mit einer Delokalisierung der negativen Ladung über $\mathrm{O}(1)$ und $\mathrm{C}(1)$ konsistent; sowohl im Anion von $1 \mathbf{a}$ als auch im Anion von 2a wird die Struktur durch die (-I)-Substituenten stabilisiert.

Die Arbeit wurde mit Lottomitteln des Landes Niedersachsen unterstützt.

\footnotetext{
* Weitere Einzelheiten zur Kristallstrukturuntersuchung können beim Fachinformationszentrum Energie, Physik, Mathematik GmbH, D-7514 Eggenstein-Leopoldshafen 2, unter Angabe der Hinterlegungsnummer CSD 51447 des Autors und des Zeitschriftenzitates angefordert werden.
}

[1] H. W. Roesky, H. Djarrah, J. Lucas, M. Noltemeyer und G. M. Sheldrick, Angew. Chem. 95, 1029 (1983).

[2] H. W. Roesky, J. Lucas, K. Keller, K. S. Dhathathreyan, M. Noltemeyer und G. M. Sheldrick, Chem. Ber., im Druck.

[3] D. W. Wiley, O. W. Webster und E. P. Blanchard, J. Org. Chem. 41, 1889 (1976).
[4] H. W. Roesky, H. Hofmann, W. Pinkert, P. G. Jones und G. M. Sheldrick, unveröffentlicht.

[5] W. R. Carpenter und G. J. Palenik, J. Org. Chem. 32, 1219 (1967).

[6] G. J. Palenik, Acta Crystallogr. 20, 471 (1966).

[7] W. Clegg, Acta Crystallogr. A 37, 22 (1981). 


\section{Zur Kenntnis von Oligophosphidosilikaten(IV) und -germanaten(IV): $\mathrm{Na}_{10} \mathrm{Si}_{2} \mathbf{P}_{6}$ und $\mathrm{Na}_{10} \mathrm{Ge}_{2} \mathbf{P}_{6}$}

On Oligophosphidosilicates(IV) and -germanates(IV): $\mathrm{Na}_{10} \mathrm{Si}_{2} \mathrm{P}_{6}$ and $\mathrm{Na}_{10} \mathrm{Ge}_{2} \mathrm{P}_{6}$

Brigitte Eisenmann*

Abt. II für Anorganische Chemie im E-Zintl-Institut der TH Darmstadt, Hochschulstraße 4, D-6100 Darmstadt

und

Mehmet Somer

Anorganisch-Chemisches Institut der TU Clausthal, Paul-Ernst-Straße 4, D-3392 Clausthal-Zellerfeld

Z. Naturforsch. 40b, 886-890 (1985); eingegangen am 28. Februar 1985

Oligophosphidosilicates(IV), Oligophosphidogermanates(IV), Synthesis, Crystal Structure

The new compounds $\mathrm{Na}_{10} \mathrm{Si}_{2} \mathrm{P}_{6}$ and $\mathrm{Na}_{10} \mathrm{Ge}_{2} \mathrm{P}_{6}$ crystallize in the monoclinic system (space group: $\mathrm{P} 2_{1} / n$ (No. 14)) with the lattice constants:

$\mathrm{Na}_{10} \mathrm{Si}_{2} \mathrm{P}_{6}: a=1312.5(5) \mathrm{pm}, b=732.5(3) \mathrm{pm}, c=791.5(3) \mathrm{pm}, \beta=90.65(7)^{\circ}$.

$\mathrm{Na}_{10} \mathrm{Ge}_{2} \mathrm{P}_{6}: a=1317.6(5) \mathrm{pm}, b=736.4(3) \mathrm{pm}, c=804.2(3) \mathrm{pm}, \beta=90.26(7)^{\circ}$.

Two $\mathrm{SiP}_{4}-$ and $\mathrm{GeP}_{4}$-tetrahedra, resp., are connected by a common edge to form $\mathrm{Si}_{2} \mathrm{P}_{6}{ }^{10-}$ and $\mathrm{Ge}_{2} \mathrm{P}_{6}{ }^{10-}$ anions.

Vor kurzem wurde gezeigt, daß in den isolierten $\mathrm{SiO}_{4}{ }^{4-}$ - bzw. $\mathrm{GeO}_{4}{ }^{4-}$-Anionen der ortho-Oxosilikate und -germanate die $\mathrm{O}^{2-}$-Ionen durch $\mathrm{P}^{3-}$-Teilchen ersetzt sein können [1]. Es resultieren $\mathrm{SiP}_{4}{ }^{8-}$ - bzw. $\mathrm{GeP}_{4}{ }^{8-}$-Anionen, die z.B. im Phosphidosilikat $\mathrm{Ba}_{4} \mathrm{SiP}_{4}$ bzw. -germanat $\mathrm{Ba}_{4} \mathrm{GeP}_{4}$ nachgewiesen wurden. Diese Anionen können über gemeinsame Kanten kondensieren, wobei, wie im Fall des $\mathrm{K}_{2} \mathrm{SiP}_{2}$ gezeigt [2], unendliche $\left(\mathrm{SiP}_{2}{ }^{2-}\right)_{\infty}$-Kettenanionen entstehen, die strukturell völlig den dazu isosteren neutralen $\mathrm{SiS}_{2}$-Molekülen entsprechen. Im $\mathrm{Na}_{10} \mathrm{Si}_{2} \mathrm{P}_{6}$ und $\mathrm{Na}_{10} \mathrm{Ge}_{2} \mathrm{P}_{6}$ gelang es uns jetzt, eine Zwischenstufe dieser Vernetzung zu isolieren.

\section{Ergebnisse und Diskussion}

\section{Darstellungen}

Beide Verbindungen wurden direkt aus den Elementen synthetisiert. Da bei einem stöchiometrischen Ansatz die Reaktionsprodukte mit Natriumphosphiden verunreinigt waren, wurden diese Elemente im Überschuß eingesetzt. Gute Ergebnisse wurden erzielt, wenn die Elemente im Atomverhältnis $\mathrm{Na}: \mathrm{Ge}: \mathrm{P}=5: 1: 3$ vorgegeben wurden. Als Gefäßmaterial dienten verschweißte Eisentiegel, die in Quarzampullen eingeschmolzen waren. Die Proben

\footnotetext{
* Sonderdruckanforderungen an Dr. B. Eisenmann.
}

Verlag der Zeitschrift für Naturforschung, D-7400 Tübingen 0340-5087/85/0700-0886/\$01.00/0 wurden innerhalb von 6 Stunden auf $850{ }^{\circ} \mathrm{C}$ erhitzt und innerhalb 24 Stunden auf Raumtemperatur abgekühlt. Wegen der extremen Empfindlichkeit gegen feuchte Luft wurden die Verbindungen in einem Handschuhkasten unter Inertgas gehandhabt. In beiden Fällen entstanden beigefarbene Reguli, aus denen durchsichtige leistenförmige Kristalle abgespalten werden konnten. Die Stöchiometrie beider Verbindungen stützt sich auf vollständige Röntgenstrukturanalysen mit Einkristalldaten.

\section{Strukturbestimmung}

Bereits die Pulveraufnahmen zeigten, daß beide Verbindungen einander isotyp sind. Zur Strukturbestimmung wurde jeweils ein leistenförmiger Einkristall, mit getrocknetem, schwerem Paraffinöl bedeckt, in eine vorgetrocknete Lindemannkapillare eingeschmolzen. Nach Drehkristall- und Weißenbergaufnahmen (CuK $\alpha$-Strahlung) kristallisieren beide Verbindungen monoklin mit der zonalen Interferenzbedingung: Reflexe $h 0 l$ nur vorhanden für $h+l=2 n$. Zusätzlich wurde noch die seriale Bedingung: Reflexe $0 k 0$ nur vorhanden für $k=2 n$ beobachtet. Damit ist allein die Raumgruppe $\mathrm{P} 2_{1} / n$ verträglich. Zur Bestimmung der Gitterkonstanten wurden an einem automatischen Vierkreisdiffraktometer (Philips PW 1100, MoK $\alpha$, Graphitmonochromator) jeweils 25 ausgewählte Reflexe genau zentriert und aus den Winkelwerten die Elementarzellpara- 
Tab. I. Die kristallographischen Daten der Verbindungen $\mathrm{Na}_{10} \mathrm{Ge}_{2} \mathrm{P}_{6}$ und $\mathrm{Na}_{10} \mathrm{Si}_{2} \mathrm{P}_{6}$. (Standardabweichungen in Klammern, U-Werte in $\mathrm{pm}^{2}$.)

Der anisotrope Temperaturfaktor ist definiert als

$\exp \left[-2 \pi^{2}\left(h^{2} a^{* 2} \mathrm{U}_{11}+k^{2} b^{* 2} \mathrm{U}_{22}+l^{2} c^{* 2} \mathrm{U}_{33}+2 h k a^{*} b^{*} \mathrm{U}_{12}+2 h l a^{*} c^{*} \mathrm{U}_{13}+2 k l b^{*} c^{*} \mathrm{U}_{23}\right)\right]$.

\begin{tabular}{lcc}
\hline & $\mathrm{Na}_{10} \mathrm{Ge}_{2} \mathrm{P}_{6}$ & $\mathrm{Na}_{10} \mathrm{Si}_{2} \mathrm{P}_{6}$ \\
\hline Kristallsystem und Raumgruppe: & $\mathrm{P} 2{ }_{1} / n$, monoklin $(\mathrm{Nr} .14)$ & \\
Gitterkonstanten $(\mathrm{pm}) a:$ & $1317,6(5)$ & $1312,5(5)$ \\
$b:$ & $736,4(3)$ & $732,5(3)$ \\
$c:$ & $804,2(3)$ & $791,5(3)$ \\
$\beta:$ & $90,26(7)^{\circ}$ & $90,65(7)^{\circ}$ \\
Zahl der Formeleinheiten: & 2 & 2 \\
Volumen der EZ $\left(\mathrm{pm}^{3}\right):$ & $780,29 \cdot 10^{6}$ & $760,9 \cdot 10^{6}$ \\
$\mu(\mathrm{MoK} \alpha)\left(\mathrm{cm}^{-1}\right):$ & 48,95 & 10,63 \\
$\varrho$ rö $\left(\mathrm{g} / \mathrm{cm}^{3}\right)$ & $2,38_{7}$ & $2,05_{9}$
\end{tabular}

Quelle der zur Rechnung verwendeten Atomformfaktoren: Internationale Tabellen

Atomparameter (alle Atome auf der Lage $4 \mathrm{e}$ mit $x, y, z ; 1 / 2-x, 1 / 2+y, 1 / 2-z ; \bar{x}, \bar{y}, \bar{z} ; 1 / 2+x, 1 / 2-y, 1 / 2+z$ )

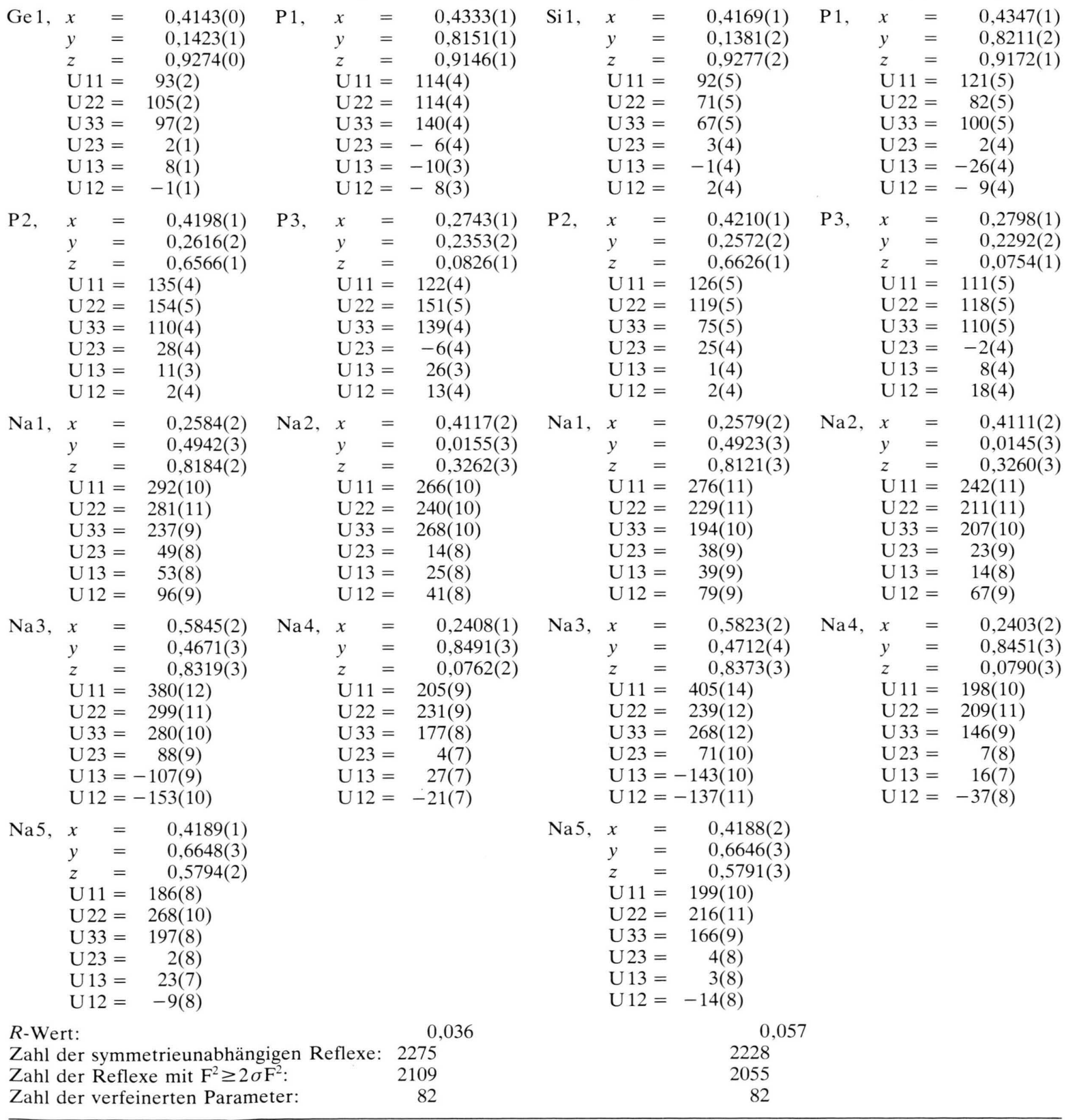


meter nach der Methode der kleinsten Fehlerquadrate optimiert (Tab. I). Zur Bestimmung der Atompositionen wurden im Winkelbereich $5^{\circ} \leq 2 \vartheta \leq 60^{\circ}$ die Intensitäten von $3567\left(\mathrm{Na}_{10} \mathrm{Ge}_{2} \mathrm{P}_{6}\right)$ bzw. 4758 $\left(\mathrm{Na}_{10} \mathrm{Si}_{2} \mathrm{P}_{6}\right)$ Reflexen vermessen $(\omega / 2 \vartheta$-scan $)$. Nach den üblichen winkelabhängigen Korrekturen und Mittelung über symmetrieabhängige Reflexe verblieben 2275 bzw. 2229 Meßwerte, von denen 166 bzw. $172<2 \sigma \mathrm{F}^{2}$ waren. Die Lösung der Struktur gelang über statistische Phasenbestimmungsmethoden (Programmsystem Multan 78 [3] in Verbindung mit XMY 84 [4]). Die erhaltenen Atompositionen einschließlich anisotrop aufgespaltener Temperaturfaktoren wurden nach der Methode der kleinsten Fehlerquadrate optimiert [5] (Tab. I). Auf eine Absorptionsund Extinktionskorrektur wurde verzichtet.

\section{Strukturbeschreibung}

Die Strukturen beider Verbindungen sind durch verzerrte $\mathrm{SiP}_{4}$ - bzw. $\mathrm{GeP}_{4}$-Tetraeder charakterisiert, die über eine gemeinsame Kante zu Doppeltetraedern verknüpft sind. Ihre Anordnung in der Zelle ist in Abb. 1 dargestellt. Die SiP- bzw. GeP-Abstände zu den terminalen P-Atomen sind mit 225,8 pm und 227,4 pm bzw. 233,4 pm und 235,1 pm deutlich kürzer als zu den verbrückenden P-Atomen mit
$230,9 \mathrm{pm}$ und $233,5 \mathrm{pm}$ bzw. 239,1 pm und $242,5 \mathrm{pm}$. Von den Bindungswinkeln an den Element(IV)atomen ist derjenige zwischen den verknüpfenden P-Atomen (93, $6^{\circ}$ im Fall der Si-, 93, $8^{\circ}$ im Fall der Ge-Verbindung) am kleinsten. Dieser Befund ist zusammen mit den aufgeweiteten Bindungslängen zu den Brückenatomen typisch für kantenverknüpfte Polyeder, deren Zentralatome keine bindende Wechselwirkung untereinander zeigen. Trotz dieser Aufweitung betragen die Bindungswinkel an den P-Atomen des Vierrings nur $86,4^{\circ}$ bzw. $86,2^{\circ}$. Die $\mathrm{Si}_{2} \mathrm{P}_{6}$ - bzw. $\mathrm{Ge}_{2} \mathrm{P}_{6}$-Anionen sind wie die Abb. 1 zeigt, durch Na-Ionen voneinander getrennt, die dabei sehr unregelmäßig von P-, Si- bzw. GeAtomen und weiteren gleichnamigen Nachbarn umgeben sind (Tab. II). Der kleinste Na-Na-Abstand beträgt im Falle der Si-Verbindung 308,9 pm bzw. im Fall der Ge-Verbindung 312,7 pm.

\section{Diskussion}

Mit den hier aufgefundenen $\mathrm{Si}_{2} \mathrm{P}_{6}{ }^{10-}$ - bzw. $\mathrm{Ge}_{2} \mathrm{P}_{6}{ }^{10-}$-Anionen wird eine Lücke in der Reihe isosterer analog gebauter Spezies geschlossen (Tab. III). Diese beginnt bei den neutralen $\mathrm{Al}_{2} \mathrm{Br}_{6}$ bzw. $\mathrm{Si}_{2} \mathrm{~S}_{2} \mathrm{Br}_{4}$-Molekülen. Das isostere, relativ niedrig geladene $\mathrm{P}_{2} \mathrm{~S}_{6}{ }^{2-}$-Anion wurde erst kürzlich z.B.

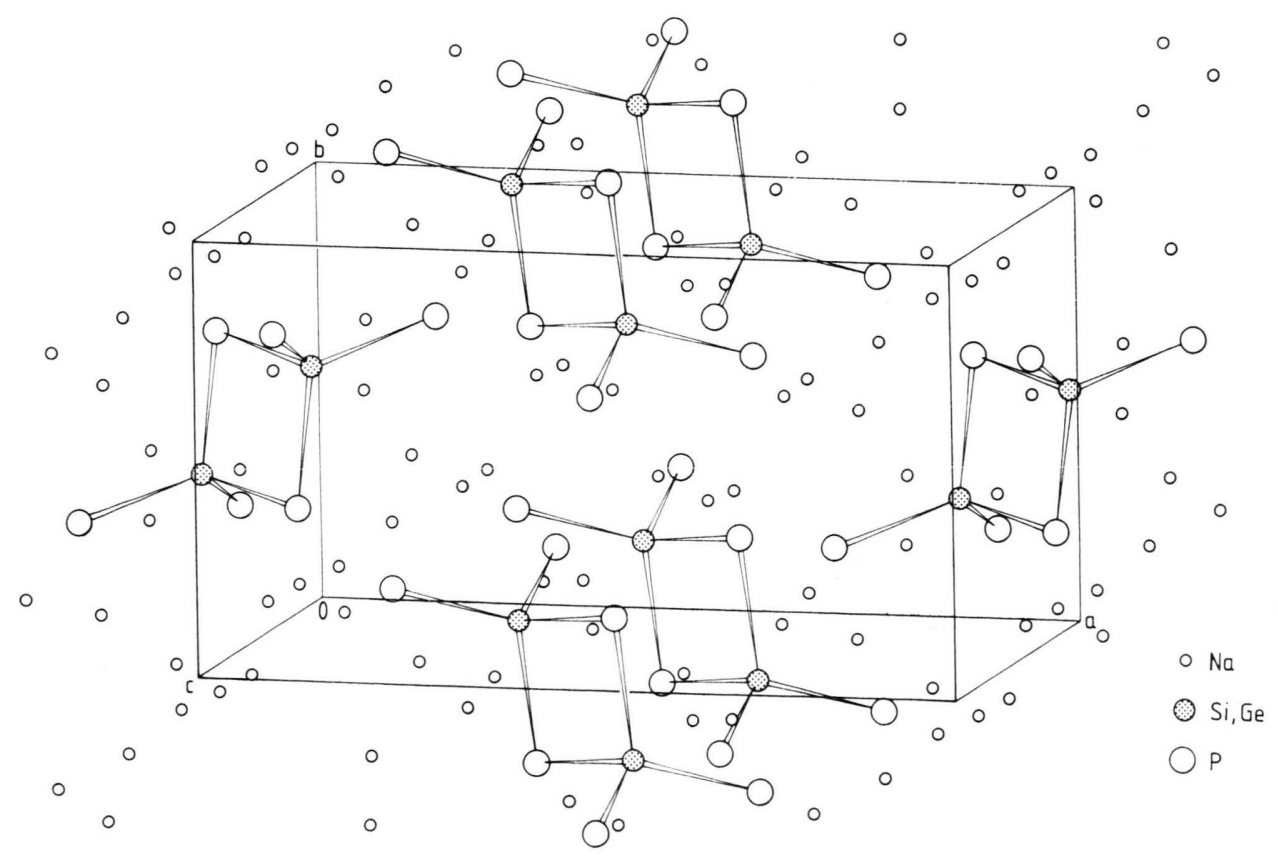

Abb. 1. Perspektivische Darstellung der Struktur des $\mathrm{Na}_{10} \mathrm{Si}_{2} \mathrm{P}_{6}$ bzw. $\mathrm{Na}_{10} \mathrm{Ge}_{2} \mathrm{P}_{6}$. 


\begin{tabular}{cccccc}
\hline $\mathrm{Na}_{10} \mathrm{Ge}_{2} \mathrm{P}_{6}$ & \multicolumn{5}{c}{$\mathrm{Na}_{10} \mathrm{Si}_{2} \mathrm{P}_{6}$} \\
$\mathrm{Ge} 1-\mathrm{P} 3$ & 233,4 & $\mathrm{KZ}=4$ & $\mathrm{Si} 1-\mathrm{P} 3$ & 225,8 \\
$-\mathrm{P} 2$ & 235,1 & $-\mathrm{P} 2$ & 227,4 & $\mathrm{KZ}=4$ \\
$-\mathrm{P} 1$ & 239,1 & & 230,9 & \\
$-\mathrm{P} 1$ & 242,5 & & $\mathrm{P} 1$ & 233,5 \\
$\mathrm{P} 3-\mathrm{Ge} 1-\mathrm{P} 2$ & $114,4^{\circ}$ & & $\mathrm{P} 3-\mathrm{Si} 1-\mathrm{P} 2$ & $113,1^{\circ}$ & \\
$\mathrm{P} 3-\mathrm{Ge} 1-\mathrm{P} 1$ & $110,0^{\circ}$ & & $\mathrm{P} 3-\mathrm{Si} 1-\mathrm{P} 1$ & $111,0^{\circ}$ & \\
$\mathrm{P} 3-\mathrm{Ge} 1-\mathrm{P} 1$ & $113,4^{\circ}$ & & $\mathrm{P} 3-\mathrm{Si} 1-\mathrm{P} 1$ & $113,1^{\circ}$ & \\
$\mathrm{P} 2-\mathrm{Ge} 1-\mathrm{P} 1$ & $114,4^{\circ}$ & & $\mathrm{P} 2-\mathrm{Si} 1-\mathrm{P} 1$ & $114,4^{\circ}$ & \\
$\mathrm{P} 2-\mathrm{Ge} 1-\mathrm{P} 1$ & $109,2^{\circ}$ & & $\mathrm{P} 2-\mathrm{Si} 1-\mathrm{P} 1$ & $110,2^{\circ}$ & \\
$\mathrm{P} 1-\mathrm{Ge} 1-\mathrm{P} 1$ & $93,8^{\circ}$ & & $\mathrm{P} 1-\mathrm{Si} 1-\mathrm{P} 1$ & $93,6^{\circ}$ & \\
$\mathrm{Na} 1-\mathrm{P}$ & $286,3-339,0$ & $\mathrm{KZ}=5$ & $\mathrm{Na} 1-\mathrm{P}$ & $285,0-334,0$ & $\mathrm{KZ}=5$ \\
$-\mathrm{Ge}$ & 319,5 & & $-\mathrm{Si}$ & 314,8 & \\
$\mathrm{Na} 2-\mathrm{P}$ & $301,7-328,7$ & $\mathrm{KZ}=7$ & $\mathrm{Na} 2-\mathrm{P}$ & $297,0-336,1$ & $\mathrm{KZ}=7$ \\
$-\mathrm{Ge}$ & $324,8-334,2$ & & $-\mathrm{Si}$ & $323,8-328,2$ & \\
$\mathrm{Na} 3-\mathrm{P}$ & $292,0-332,1$ & $\mathrm{KZ}=5$ & $\mathrm{Na} 3-\mathrm{P}$ & $290,2-327,9$ & $\mathrm{KZ}=5$ \\
$-\mathrm{Ge}$ & 337,1 & $-\mathrm{Si}$ & 334,8 & \\
$\mathrm{Na} 4-\mathrm{P}$ & $286,6-289,1$ & $\mathrm{KZ}=5$ & $\mathrm{Na} 4-\mathrm{P}$ & $286,1-290,8$ & $\mathrm{KZ}=5$ \\
$-\mathrm{Ge}$ & 336,8 & & $-\mathrm{Si}$ & 338,8 & $\mathrm{KZ}=4$ \\
$\mathrm{Na} 5-\mathrm{P}$ & $290,1-303,4$ & $\mathrm{KZ}=4$ & $\mathrm{Na} 5-\mathrm{P}$ & $290,6-305,6$ & $\mathrm{~K}$ \\
\hline & & & & &
\end{tabular}

Tab. II. Atomabstände (pm) und wichtige Bindungswinkel $\left(^{\circ}\right)$ im $\mathrm{Na}_{10} \mathrm{Ge}_{2} \mathrm{P}_{6}$ und $\mathrm{Na}_{10} \mathrm{Si}_{2} \mathrm{P}_{6}$. Die maximalen Standardabweichungen betragen im Fall des $\mathrm{Na}_{10} \mathrm{Ge}_{2} \mathrm{P}_{6}$ für die Abstände $\mathrm{Ge}-\mathrm{P} \quad 0,1 \mathrm{pm}$, $\mathrm{Na}-\mathrm{P} 0,3 \mathrm{pm}$, und die Winkelangaben $<0,1^{\circ}$, im Fall des $\mathrm{Na}_{10} \mathrm{Si}_{2} \mathrm{P}_{6}$ für die $\mathrm{Ab}$ stände $\mathrm{Si}-\mathrm{P} 0,2 \mathrm{pm}, \mathrm{Na}-\mathrm{P}$ $0,3 \mathrm{pm}$, und die Winkelangaben $<0,1^{\circ}$.

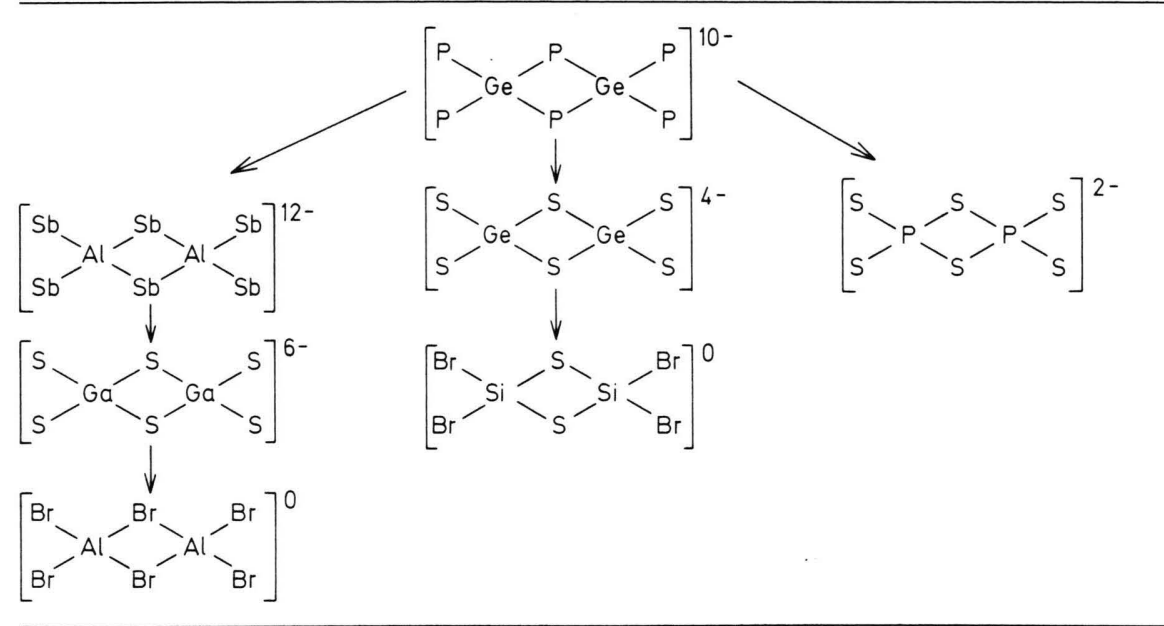

Tab. III. Beispiele für den Isosteriebezug der $\left[\mathrm{Si}_{2} \mathrm{P}_{6}\right]^{10-}$ - bzw. $\left[\mathrm{Ge}_{2} \mathrm{P}_{6}\right]^{10-}$ Anionen $\mathrm{zu}$ anderen $\mathrm{B}_{2} \mathrm{X}_{6}$ Anionen bzw. Molekülen. (Zwei $\mathrm{BX}_{4}$-Tetraeder sind über eine gemeinsame Kante zu einer zweikernigen Einheit verknüpft.) im $\mathrm{KPS}_{3}$ strukturell nachgewiesen [6]. Auch die $\mathrm{Ge}_{2} \mathrm{~S}_{6}{ }^{4-}$ - [7] und $\mathrm{Ge}_{2} \mathrm{~S}_{6}{ }^{6-}$-Anionen [8] sind durch röntgenographische Strukturbestimmungen entsprechender Verbindungen belegt. Über die hier beschriebenen $\mathrm{Si}_{2} \mathrm{P}_{6}{ }^{10-}$ - und $\mathrm{Ge}_{2} \mathrm{P}_{6}{ }^{10-}$-Ionen hinaus wurden vor kurzem sogar die hoch geladenen $\mathrm{Al}_{2} \mathrm{Sb}_{6}{ }^{12-}$-Anionen in metallisch silberhell glänzenden Zintlphasen, z. B. im $\mathrm{Ba}_{6} \mathrm{Al}_{2} \mathrm{Sb}_{6}$, nachgewiesen [9]. Diese Reihe demonstriert erneut die elementare Bedeutung, die dem Begriff der Isosterie in der
Strukturchemie der Verbindungen der Hauptgruppenelemente zukommt.

Herrn Prof. Dr. Herbert Schäfer danken wir sehr herzlich für seine Unterstützung und anregenden Diskussionen.

Der Deutschen Forschungsgemeinschaft danken wir für ihre finanzielle Unterstützung, Frau Vera Klink für ihre Mithilfe bei den kristallographischen Rechnungen. 
[1] B. Eisenmann, H. Jordan und H. Schäfer, Angew. Chem. 93, 211 (1981); Z. Anorg. Allg. Chem. 475, 74 (1981); Mat. Res. Bull. 17, 95 (1982).

[2] B. Eisenmann und M. Somer, Z. Naturforsch. 39b, 736 (1984).

[3] Multan 78, A system of computer programs, P. Main, S. E. Hull, L. Lessinger, G. Germain, J. P. Declerq und M. M. Woolfson, University of York, York, England (1983).

[4] T. Debaerdemaeker und M. M. Woolfson, Acta Crystallogr. A39, 193 (1983).
[5] G. Sheldrick, Programmsystem SHELX-76, Univ. Cambridge, Cambridge, England (1976).

[6] W. Brockner, R. Becker, B. Eisenmann und H. Schäfer, Z. Anorg. Allg. Chem. 520, 51 (1985).

[7] B. Krebs, S. Pohl und W. Schiwy, Z. Anorg. Allg. Chem. 393, 241 (1972).

[8] B. Eisenmann, M. Jakowski und H. Schäfer, Rev. Chim. Minéral. 21, 12 (1984).

[9] G. Cordier, G. Savelsberg und H. Schäfer, Z. Naturforsch. 37 b, 975 (1982). 